\title{
Effect of a rye B chromosome and its segments on homoeologous pairing in hybrids between common wheat and Aegilops variabilis
}

\author{
Ryota Kousaka and Takashi R. Endo* \\ Laboratory of Plant Genetics, Graduate School of Agriculture, \\ Kyoto University, Kyoto 606-8502, Japan
}

(Received 6 December 2011, accepted 20 January 2012)

\begin{abstract}
Rye B chromosomes, which are supernumerary chromosomes dispensable for the host but increase in number by non-disjunction after meiosis, have been reported to affect meiotic homoeologous pairing in wheat-rye hybrids. The effect of a rye B chromosome (B) and its segments (B-9 and B-10) on homoeologous pairing was studied in hybrids between common wheat $(2 \mathrm{n}=42)$ and Aegilops variabilis $(2 \mathrm{n}=$ 28 ), with reference to the $P h 1$ gene located on wheat chromosome $5 \mathrm{~B}$. The B-9 and B-10 chromosomes are derived from reciprocal translocations between a wheat and the B chromosomes, and the former had the B pericentromeric segment and the latter had the B distal segment. Both the B and B-9 chromosomes suppressed homoeologous pairing when chromosome $5 \mathrm{~B}$ was absent. On the other hand, the B-9 and B-10 chromosomes promoted homoeologous pairing when 5B was present. On pairing suppression, B-9 had a greater effect in one dose than in two doses, and B-9 had a greater effect than B-10 had in one dose. These results suggested that the effect of the $\mathrm{B}$ chromosomes on homoeologous pairing was not confined to a specific region and that the intensity of the effect varied depending on the presence or absence of $5 \mathrm{~B}$ and also on the segment and dose of the $\mathrm{B}$ chromosome. The mean chiasma frequency $(10.23)$ in a hybrid $(2 \mathrm{n}=36)$ possessing $5 \mathrm{~B}$ and one B-9 was considerably higher than that $(2.78)$ of a hybrid $(2 \mathrm{n}=35)$ possessing $5 \mathrm{~B}$ alone, and was comparable with that (14.09) of a hybrid $(2 \mathrm{n}=34)$ lacking 5B. This fact suggested that the B chromosome or its segment can be used in introducing alien genes into wheat by inducing homoeologous pairing between wheat and alien chromosome.
\end{abstract}

Key words: homoeologous pairing, $P h 1, \mathrm{~B}$ chromosome, rye, common wheat

\section{INTRODUCTION}

One of the wheat breeding approaches is to introduce agronomically useful traits such as resistance to rust from wild relative species (Chhuneja et al., 2008). This can be achieved by interspecific hybridization between wheat and related species, followed by successive backcrossing of the hybrids to wheat. Thus, useful traits of wild species can be introduced into wheat lines carrying single chromosomes of wild species, i.e. alien chromosome addition or substitution lines. In fact, many alien chromosome addition lines of wheat have been established for the breeding purpose (Jiang et al., 1993; Kang et al., 2011). However, when a whole alien chromosome is introduced into wheat, not only beneficial traits but also

Edited by Minoru Murata

* Corresponding author. E-mail: endo.takashi.2e@kyoto-u.ac.jp undesirable ones are naturally introduced from alien species. This problem can partially be solved by using radiation to transfer small alien chromosome segments to wheat chromosomes (Sears and Gustafson, 1993) or by causing homoeologous pairing between wheat and alien chromosomes (Riley et al., 1968; Lukaszewski, 2000). Although the wheat chromosomes have a potential to pair and recombine with alien homoeologous chromosomes, the $P h 1$ gene on a wheat chromosome 5B restricts chromosome pairing to homologues by suppressing homoeologous pairing (Riley and Chapman, 1958). Therefore, when $P h 1$ is absent, homoeologous pairing and recombination occur between the wheat and alien chromosomes, and genes on the alien chromosomes can be introduced into wheat. Useful though $P h 1$ is in inducing homoeologous pairing, it takes two crosses between an alien substitution line and a line nullisomic for $5 \mathrm{~B}$ (nullisomic 5B-tetrasomic 5A or 5D) or a Ph mutant line (Sears, 1977) 
to produce a line carrying an alien chromosome and no Ph1. Moreover, a cytological or molecular analysis is necessary to screen for the critical plants.

Utilization of B chromosomes can be another approach to inducing homoeologous pairing in wheat. B chromosomes are dispensable for the development of its host, increase in number by non-disjunction, and found within many plants and animals (Jones et al., 2008). Many studies have been conducted on the effect of rye B chromosomes on pairing between homoeologous chromosomes in wheat-rye hybrids, but somewhat inconsistent results have been reported in those studies (Jones and Puertas, 1993). Viegas (1980) reported that B chromosomes of rye suppress homoeologous pairing. However, Roothaan and Sybenga (1976) reported that rye B chromosome have no effect on homoeologous pairing. Romero and Lacadena (1980) reported that when chromosome 5B is present, rye B chromosomes promote homoeologous pairing and that, in contrast, when chromosome 5B is absent, rye B chromosomes suppress homoeologous pairing. This result implies that homoeologous pairing is affected by $\mathrm{B}$ Ph1 interaction.

One of the objectives of the present study was to reveal what kind of effect a rye B chromosome has on homoeologous pairing in reference to the presence or absence of the Ph1 gene in hybrids between common wheat and Aegilops variabilis. The other objective was to determine which region of the rye $B$ chromosome had a factor controlling homoeologous pairing. The latter study was conducted using the dissection lines of the rye B chromosome, i.e. common wheat lines carrying different segments of the rye B chromosome (Endo et al., 2008).

\section{MATERIALS AND METHODS}

Plant materials An aneuploid line nullisomic 5Btetrasomic 5D (four doses of chromosome 5D compensate for the lack of chromosome 5B) of a common wheat cultivar Chinese Spring (Triticum aestivum, $2 \mathrm{n}=6 \mathrm{x}=42$, genome formula AABBDD) was crossed with a Chinese Spring line carrying a whole rye B chromosome (abbreviated as B in this paper) and also with a Chinese Spring line carrying reciprocal translocations between the rye $\mathrm{B}$ chromosome and a wheat chromosome (abbreviated as B9 and B-10 in this paper). The lines carrying the rye B and two translocation chromosomes were developed by Endo et al. (2008) (they designated B, B-9 and B-10 as B , $B^{\mathrm{s}}-9$ and $B^{\mathrm{s}}-10$, respectively in their paper). From the former cross, a hybrid $(2 \mathrm{n}=44)$ that was monosomic for $5 \mathrm{~B}$, trisomic for 5D and disomic for $\mathrm{B}$ was obtained, and from the latter, a hybrid $(2 \mathrm{n}=46)$ that was monosomic for $5 \mathrm{~B}$, trisomic for $5 \mathrm{D}$, trisomic for $\mathrm{B}-9$, and monosomic for B-10 was obtained. These hybrids were pollinated with Ae. variabilis $(2 \mathrm{n}=4 \mathrm{x}=28$, UUSS). Hybrid plants with the following chromosome constitutions were used in this study: $2 \mathrm{n}=34$ (nullisomic for $5 \mathrm{~B}$ : represented by $34-$ in the text and Table 1), $2 \mathrm{n}=36$ (nullisomic for $5 \mathrm{~B}$, disomic for B: $34+\mathrm{B}$ "), $2 \mathrm{n}=37$ (nullisomic for $5 \mathrm{~B}$, trisomic for B-9: 34+B-9"'), 2n = 35 (monosomic for 5B: 35-), 36 (monosomic for both $5 \mathrm{~B}$ and B-9: 35+B-9'), $2 \mathrm{n}=37$ (monosomic for $5 \mathrm{~B}$, disomic for B-9: 35+B-9"), $2 \mathrm{n}=36$ (monosomic for both 5B and B-10: 35+B-10'), $2 \mathrm{n}=37$ (monosomic for both $5 \mathrm{~B}$ and B-9, disomic for 5D: 36+B-9'), $2 \mathrm{n}=38$ (monosomic for 5B, disomic for both 5D and B-9: 36+B-9"). Plants with other chromosome combinations were neither obtained nor used because they were not suitable for meaningful comparison.

Identification of mitotic chromosomes The presence or absence of chromosome 5B in the hybrids was checked in mitotic metaphase cells of root tips by a 'modified Cbanding' procedure (Gill et al., 1991). The presence of the B, B-9 and B-10 chromosomes was confirmed by fluorescence in situ hybridization (FISH) and genomic in situ

Table 1. Frequencies of univalents, bivalents and multivalents, and chiasma frequencies in common wheat-Ae. variabilis hybrids with various chromosome constitutions concerning $5 \mathrm{~B}$, rye $\mathrm{B}$ and rye $\mathrm{B}$ segments

\begin{tabular}{|c|c|c|c|c|c|c|c|c|c|c|c|c|c|c|c|c|c|c|}
\hline \multirow[b]{2}{*}{ Hybrid* } & \multirow[b]{2}{*}{$2 n=$} & \multirow[b]{2}{*}{$5 B$} & \multirow{2}{*}{$\begin{array}{l}\text { No. cells } \\
\text { examined }\end{array}$} & \multirow{2}{*}{$\frac{\text { Univalent }}{\text { Range Mean }}$} & \multicolumn{2}{|c|}{ Bivalent (rod) } & \multicolumn{2}{|c|}{ Bivalent (ring) } & \multicolumn{2}{|c|}{ Trivalent } & \multicolumn{2}{|c|}{ Tetravalent } & \multicolumn{2}{|c|}{ Pentavalent } & \multicolumn{2}{|c|}{ Hexavalent } & \multicolumn{2}{|c|}{ Chiasma frequency } \\
\hline & & & & & Range & Mean & Range & Mean & Range & Mean & Range & Mean & Range & Mean & Range & Mean & Range & $\begin{array}{r}\text { Mean } \pm \\
\text { de }\end{array}$ \\
\hline 4- & 34 & & 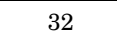 & $6-17 \quad 11.31$ & & & & & & & & & & & 0 & 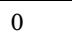 & & \\
\hline $84+8$ & 36 & sent & 64 & $7-26 \quad 16.25$ & $1-11$ & 644 & ? & 057 & 0 & 1.48 & ? & 0.19 & $0-1$ & 0.11 & $0-1$ & 0.03 & $5-17$ & $11.59 \pm 2.93$ \\
\hline $34+\mathrm{B}-9$ & 37 & bsent & 14 & $10-2417.86$ & $3-10$ & 5.86 & ? & 1.29 & $0-3$ & 1.43 & $0-2$ & 0.14 & 0 & . & 0 & 0 & $8-17$ & $11.71 \pm 2.43$ \\
\hline $35-$ & 35 & present & 41 & 20-35 29.39 & $0-7$ & 2.49 & $0-1$ & 0.02 & $0-1$ & 0.12 & $0-1$ & 0.02 & $0-1$ & 0.02 & 0 & 0 & $0-7$ & $2.78 \pm 1.29$ \\
\hline $35+\mathrm{B}-9^{\prime}$ & 36 & present & 44 & $11-2618.2$ & $1-9$ & 5.27 & $0-1$ & 0.14 & $0-4$ & 1.89 & $0-2$ & 0.18 & $0-1$ & 0.09 & 0 & 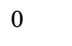 & $6-14$ & $10.23 \pm 2.28$ \\
\hline $35+B-9 "$ & 37 & present & 69 & $15-3724.09$ & $0-10$ & 5.38 & $0-1$ & 0.07 & $0-2$ & 0.58 & $0-1$ & 0.07 & $0-1$ & 0.01 & 0 & 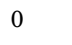 & $0-12$ & $6.96 \pm 2.08$ \\
\hline $35+\mathrm{B}-10^{\prime}$ & 36 & present & 30 & $15-3125.07$ & $1-9$ & 3.63 & $0-2$ & 0.5 & $0-5$ & 0.8 & $0-1$ & 0.07 & 0 & 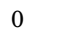 & 0 & 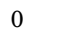 & $3-14$ & $6.43 \pm 2.45$ \\
\hline $36+\mathrm{B}-9^{\prime}$ & 37 & present & 48 & $12-3023.81$ & $1-8$ & 3.23 & $0-3$ & 1.35 & $0-3$ & 1.23 & $0-2$ & 0.08 & 0 & . & . & 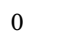 & $4-17$ & $8.65 \pm 2.53$ \\
\hline $36+\mathrm{B}-9 "$ & 38 & present & 52 & $13-2720.02$ & $3-12$ & 5.92 & $0-2$ & 0.48 & $0-3$ & 1.15 & $0-2$ & 0.25 & $0-1$ & 0.1 & $0-1$ & 0.04 & $6-14$ & $10.52 \pm 1.92$ \\
\hline
\end{tabular}

* For details of chromosome constitutions, see the text. Two plants were used for 34+B", 35+B-9' and 35+B-9", and one plant was used for the other hybrids. 
hybridization (GISH). The rye B-specific tandem repetitive sequence D1100 (Sandery et al., 1990) and the total rye genomic DNA were used as the probes of FISH and GISH, respectively. The FISH/GISH procedures were those reported by Sakai et al. (2009). Figure 1 shows the C-banding and FISH/GISH images of mitotic metaphase cells of representative plants used in this study.

Observation of meiotic pairing The screened plants were grown in a greenhouse from winter to spring. When flag leaf sheaths fully appeared, three anthers were collected from individual florets of young spikes, and the meiotic stage of pollen mother cells (PMC's) was checked by squashing one of the anthers in a drop of a $1 \%(\mathrm{w} / \mathrm{v})$ acetocarmine solution. When the PMC's of the anther were at metaphase I, the remaining two anthers were stored in a fixative (3 ethanol : 1 glacial acetic acid) for several days at room temperature and then kept in a freezer at $-20^{\circ} \mathrm{C}$ until use. The fixed anthers were stained in a $1 \%(\mathrm{w} / \mathrm{v})$ acetocarmine solution for about $1-$ $2 \mathrm{~h}$ at room temperature and preparations were made by the squash method. Briefly, the stained anther was smashed in a drop of $45 \%(\mathrm{v} / \mathrm{v})$ acetic acid with a pair of forceps, after the debris was removed, a cover slip was placed on the acetic acid solution, the slide was heated over the flame of a spirit lamp, a filter paper was placed over the cover slip, and then the slide was squashed with a finger over the filter paper. Metaphase I PMC's were observed under a phase contrast microscope and then were recorded with a digital camera, and the digital images were used to determine the meiotic configurations of the PMC's.
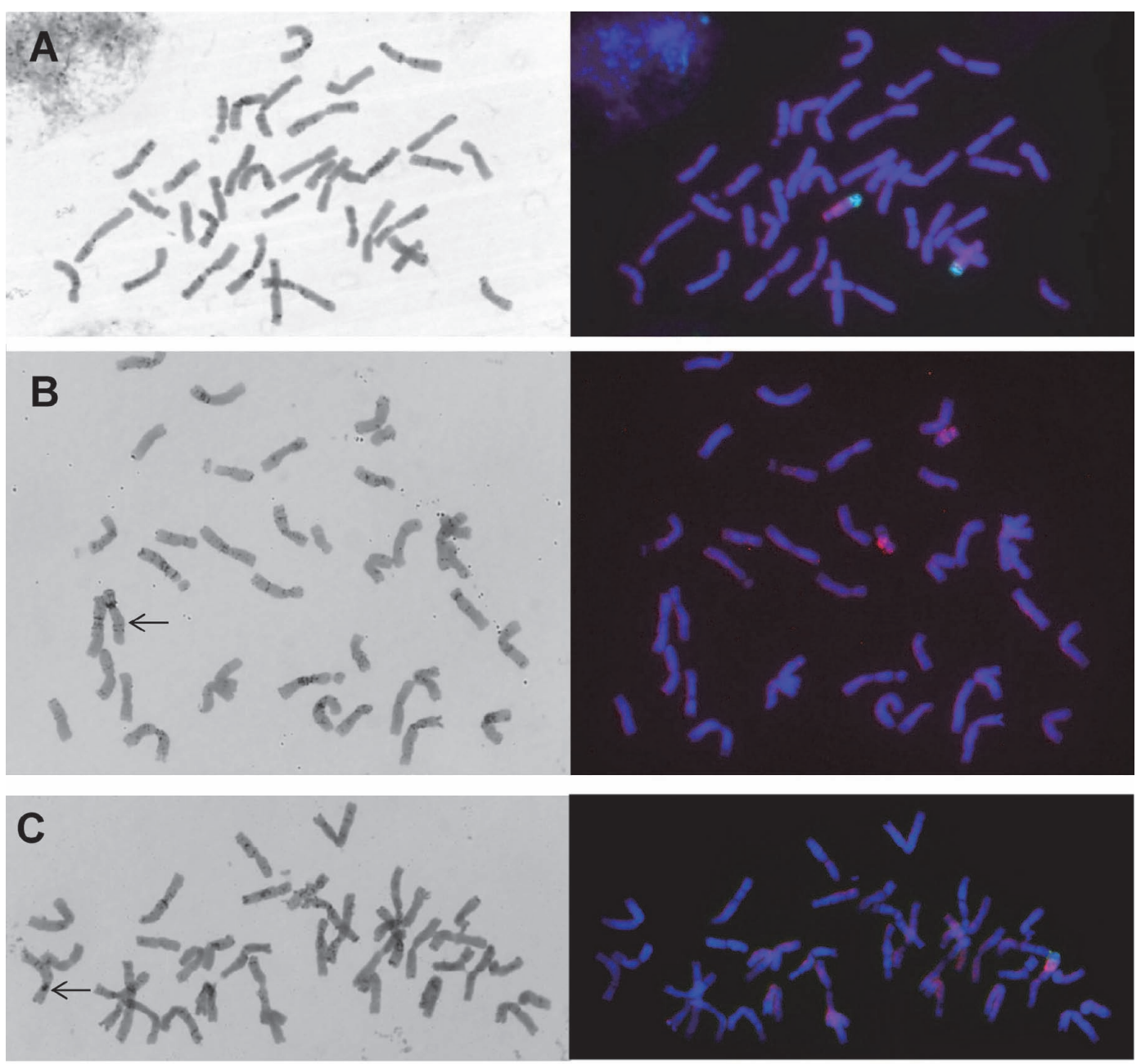

$10 \mu \mathrm{m}$

Fig. 1. Mitotic metaphase cells of representative hybrid derivatives between common wheat and Ae. variabilis. In each pair, C-banding (left) and FISH/GISH (right) were conducted with the same cells. A: 34+B", B: 35+B-9", C: 35+B-10'. Arrows indicate chromosome 5B. Note no 5B was observed in 'A'. FISH signals (green) represent the rye B specific D1100 sequence and GISH signals (red) represent rye chromatin. 


\section{RESULTS}

Chiasma frequency Meiotic configurations of PMC's were analyzed by counting the numbers of univalents, bivalents and multivalents (trivalents, tetravalents, pentavalents and hexavalents) (Fig. 2). In calculating chiasma frequency per PMC, the number of chiasmata was counted as follows: One chiasma in a rod univalent, two chiasmata in a ring bivalent and in a trivalent, three in a tetravalent, four in a pentavalent, and five in a hexavalent. The range and mean value of univalent, bivalent and multivalent, and those of chiasma frequency are summarized in Table 1. Although most of the observed bivalents and multivalents could be regarded as formed by homoeologous pairing, part of the bivalents and multivalents in some hybrids must have been formed by homologous pairing because they were disomic or trisomic for particular chromosomes and also because they carried B-9 or B-10, which had wheat chromosomal segments. Since such homologous pairings were not identifiable under a phase contrast microscope, the data in Table 1 included both homologous and homoeologous pairings.

Comparison of chiasma frequencies Chiasma frequencies between the hybrid plants were compared and statistically analyzed by Student's t-test or Welch's ttest. Representative meiotic configurations of hybrid derivatives between common wheat and Ae. variabilis are shown in Fig. 3. The details of the comparisons are described below, in due consideration of chiasmata formed by homologous pairings.

Between 34- and 35-: Among the hybrids examined, the
34- hybrid without chromosome 5B showed the lowest frequency of univalents (11.31) and the highest frequencies of trivalents (2.28) and tetravalent (0.38). Hence, the mean chiasma frequency (14.09) per PMC was the highest in all hybrids. On the other hand, the 35- hybrid with chromosome 5B showed the highest frequency of univalents and the lowest frequencies of bivalents and multivalents except pentavalents. Besides, in this hybrid, a ring bivalent was observed only in one of the PMC's and no hexavalent was observed. Consequently, the mean chiasma frequency per PMC of the 35- hybrid was the lowest (2.78) among the hybrids examined. The difference in chiasma frequency between these two hybrids, which is obviously significant (Welch's t-test, $\mathrm{p}=3.49 \mathrm{e}$ 35 ), was due to the presence or absence of the $P h 1$ gene on chromosome 5B. The comparable effect of the $P h 1$ gene on meiotic pairing was previously reported in wheatrye hybrids (Riley et al., 1959).

Between 34- and 34+B": The frequencies of univalents and multivalents of the $34+\mathrm{B}$ " hybrid overlapped those of the 34- hybrid. However, the mean chiasma frequency (11.59) was significantly lower than that of the 34- hybrid (14.09) (Welch's t-test, $p=1.67 \mathrm{e}-06$ ). Since the two B chromosomes in the $34+\mathrm{B}$ " hybrid should have formed a homologous bivalent with high frequency, the chiasma frequency of the remaining 34 chromosomes of the hybrid must have been less than 11.59. This result suggested that the two B chromosomes significantly suppressed the meiotic pairing of homoeologous chromosomes in the absence of chromosome 5B. In other words, the B chromosome had a function similar to that of the $P h 1$ gene but did not fully compensate the loss of the gene; the $P h 1$
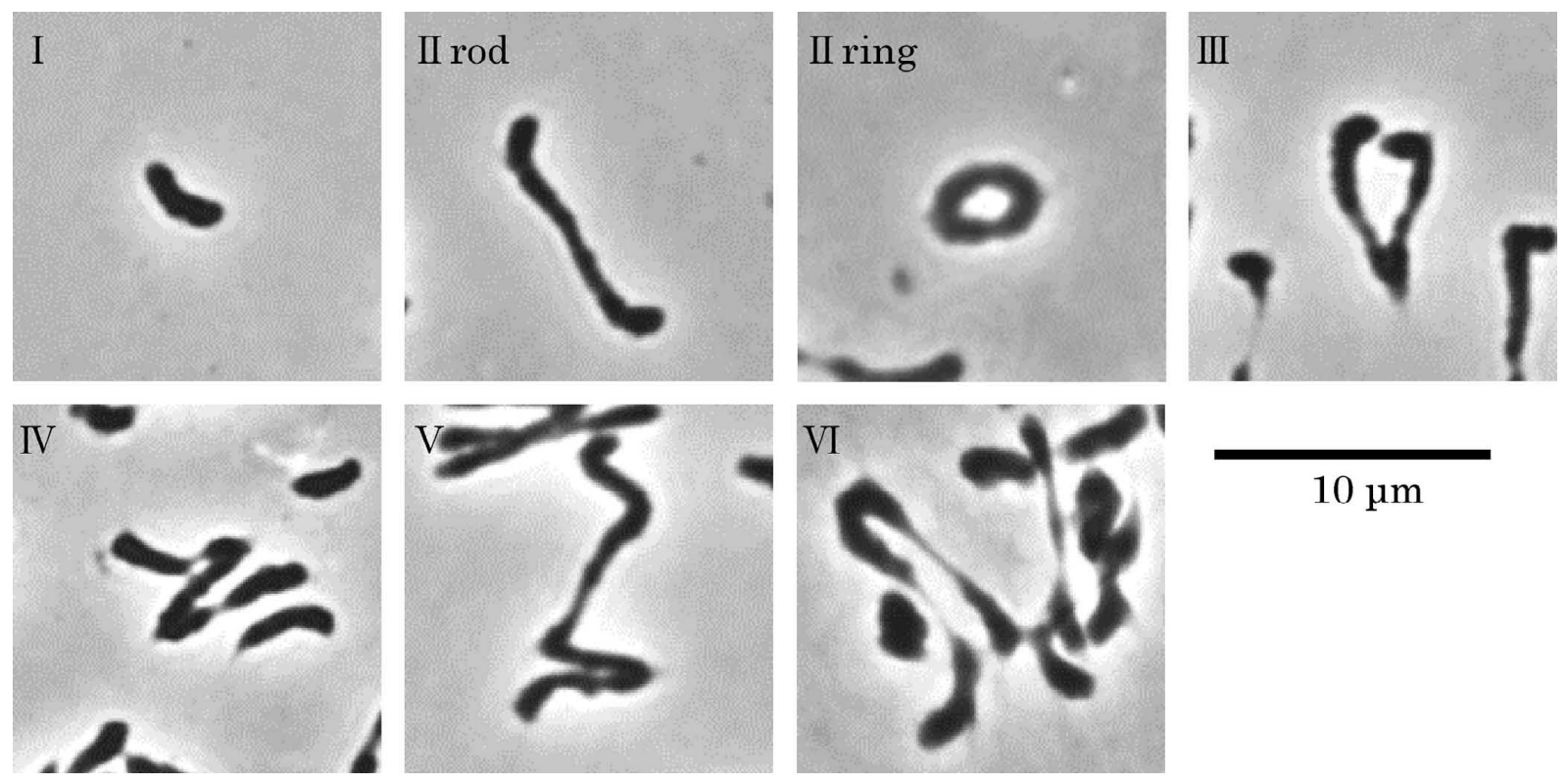

Fig. 2. Representative phase contrast images of univalent (I), rod bivalent (II rod), ring bivalent (II ring), trivalent (III), tetravalent (IV), pentavalent (V) and hexavalent (VI) at meiotic metaphase I. 

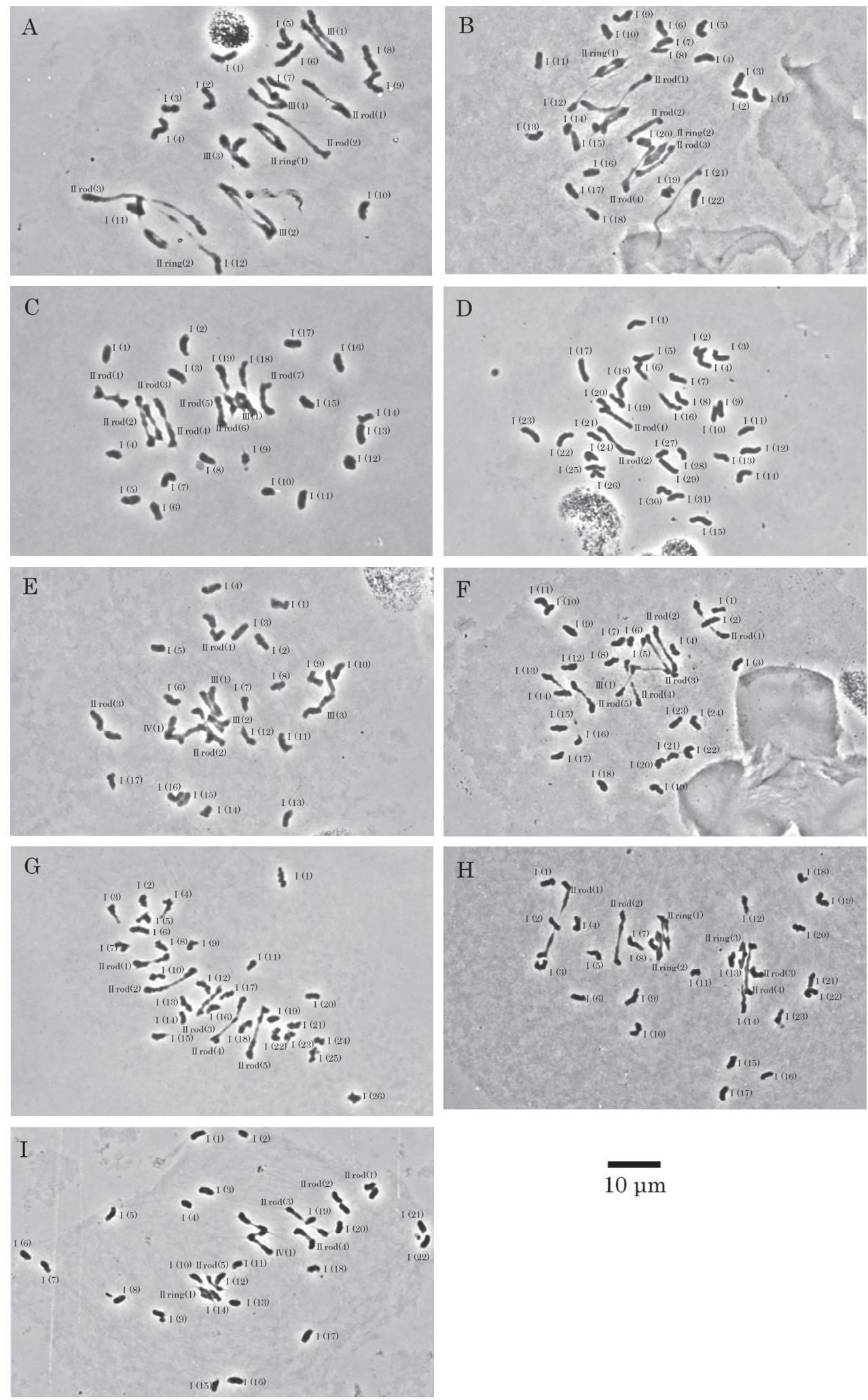

$10 \mu \mathrm{m}$

Fig. 3. Representative meiotic configurations of hybrid derivatives between common wheat and Ae. variabilis. A: 34, 10 univalents; 6 rod bivalents and 4 trivalents. B: 34+B"; 22 univalents, 4 rod bivalents; 2 ring bivalents and 1 trivalent. C: $34+\mathrm{B}-9$ "'; 18 univalents, 7 rod bivalents and 1 trivalent. D: $35-; 31$ univalents and 2 rod bivalents. E: $35+$ B-9'; 17 univalents, 3 rod bivalents, 3 trivalents and 1 tetravalent. F: $35+$ B-9"; 24 univalents, 5 rod bivalents and 1 trivalent. G: $35+$ B-10'; 26 univalents and 5 rod bivalents. H: 36+B-9'; 23 univalents, 4 rod bivalents and 3 ring bivalents. I: $36+\mathrm{B}-9$ "; 22 univalents, 5 rod bivalents, 1 ring bivalent and 1 tetravalent. 
effect was significantly stronger in suppressing homoeologous pairing (the mean chiasma frequency of the 35hybrid was 2.78) (Welch's t-test, p = 2.08 e-37).

Between 34- and 34+B-9"': The 34+B-9"' hybrid had neither pentavalent nor hexavalent, and its mean chiasma frequency (11.71) was significantly lower than that of the 34- hybrid (14.09 \pm 1.82 ) (Student's t-test, $\mathrm{p}=0.00064)$ ). Considering that the three B-9 chromosomes must have formed bivalents and multivalents among themselves or with the wheat chromosome whose segment is transferred to B-9, the chiasma frequency between homoeologous chromosomes in the 34+B-9" hybrid should be less than 11.71. This result suggested that the B-9 chromosome, like the B chromosome, a significant effect on suppressing homoeologous pairing in the absence of chromosome 5B.

Between 35- and 35+B-9': The mean frequency of every multivalent was higher by far in the $35+\mathrm{B}-9$ ' hybrid than in the 35- hybrid (both hybrids had no hexavalent). The mean chiasma frequency of the $35+\mathrm{B}-9$ ' hybrid (10.23) was significantly higher than that of the 35- hybrid (2.78) (Welch's t-test, $\mathrm{p}=1.11 \mathrm{e}-28$ ). Even if one of the 10.23 chiasmata is considered to be formed by homologous pairing between the B-9 chromosome and one of the wheat chromosomes, it is obvious that the monosomic B-9 addition raised the frequency of homoeologous pairing significantly in the presence of chromosome 5B, by suppressing the function of the $P h 1$ gene.

Between 35- and 35+B-9": The mean chiasma frequency of the 35- hybrid (2.78) was significantly lower than that of the 35+B-9" hybrid (6.96) (Welch's t-test, $\mathrm{p}=8.19 \mathrm{e}-24$ ), even if the two B-9 chromosomes in the latter hybrid are assumed to have formed a ring bivalent between themselves or a trivalent with one of the wheat chromosomes in all PMC's. In the presence of chromosome 5B, the disomic B-9 addition obviously promoted homoeologous pairing in the hybrid, but this promotion was significantly less than that caused by the monosomic addition of the B9 chromosome (10.23) (Student's t-test, $\mathrm{p}=2.71 \mathrm{e}-12$ ). This fact suggested that one dose of B-9 exerted a stronger effect on promoting homoeologous pairing than two doses of B-9 did.

Between 35- and 35+B-10': The mean chiasma frequency of the 35- hybrid (2.78) was significantly lower than that of the 35+B-10' hybrid (6.43) (Welch's t-test, $\mathrm{p}=$ 3.79 e-09). Even though the B-10 chromosome is supposed to have formed a bivalent with one of the wheat chromosomes in all PMC's, the monosomic B-10 addition raised the frequency of homoeologous pairing significantly in the presence of chromosome $5 \mathrm{~B}$, but this promotion was not as much as that by the monosomic B-9 addition (Student's t-test, $\mathrm{p}=2.34 \mathrm{e}-09$ ).

Between 36+B-9' and 36+B-9": The addition of B-9 increased chiasma frequency significantly in the $36+\mathrm{B}-9$ ' (8.65) and 36+B-9" (10.52) hybrids that were monosomic for $5 \mathrm{~B}$ and disomic for 5D. Considering the homologous pairing involving B-9, the difference in chiasma frequency between the two hybrids was not as obvious as was observed between the 35+B-9' (10.23) and 35+B-9" (6.96) hybrids that were monosomic for both $5 \mathrm{~B}$ and $5 \mathrm{D}$.

\section{DISCUSSION}

The above comparisons revealed that the whole rye B chromosome and its segments had influence on homoeologous pairing in common wheat-Ae. variabilis hybrids. This fact implied that some genetic factor affecting homoeologous pairing was not confined to a specific chromosomal region of the $\mathrm{B}$ chromosome but that there were such factors at least in the proximal and distal regions.

As previously reported in wheat-rye hybrids (Romero and Lacadena, 1980), the B chromosome and its segments had reverse effects on homoeologous pairing depending on the presence or absence of chromosome 5B, namely the Ph1 gene. When the 5B chromosome was present, B-9 and B-10 promoted homoeologous pairing. On the other hand, when chromosome 5B was absent, B and B-9 suppressed homoeologous pairing. In addition, a clear dosage effect of B-9 was demonstrated in the 35+B-9' and $35+\mathrm{B}-9$ " hybrids. In the presence of chromosome $5 \mathrm{~B}$, one dose of B-9 promoted homoeologous pairing more actively than two doses did. However, the opposite dosage effect was observed in the $36+\mathrm{B}-9$ ' and $36+\mathrm{B}-9$ " hybrids that were monosomic for $5 \mathrm{~B}$ and disomic for $5 \mathrm{D}$. This might be due to the fact that chromosome $5 \mathrm{D}$ has an effect on promoting homoeologous pairing (Feldman, 1966). Taking all these facts into consideration, the genetic factors controlling homoeologous pairing on the rye $\mathrm{B}, 5 \mathrm{~B}$ and $5 \mathrm{D}$ chromosomes seem to have similar functions and interfere with each other. This complicated interaction between the three chromosomes resembles the odd-even effect of B chromosomes in rye noticed by Jones and Rees (1969). They found that the variation in chiasma frequency of A chromosomes between PMC's increases with increasing $B$ frequency and that variances increase in an up-and-down, zig-zag fashion with increasing number of B's; variances are disproportionately high in the presence of odd as compared with even numbers. The increase of the variance in chiasma frequency can be regarded as a consequence of failure in suppressing homoeologous pairing leading to exclusive homologous pairing. In this context, the effect of the B and B segments on homoeologous pairing found in this study might be explained as follows: The rye $\mathrm{B}, 5 \mathrm{~B}$ and $5 \mathrm{D}$ chromosomes have the same function of suppressing homoeologous pairing and followed the odd-even effect.

The Ph1 locus is confined to the $2.5 \mathrm{Mb}$ region that contains a cluster of $c d k$-related genes with a large heterochromatin insertion (Griffiths et al., 2006), and the transcripts from this region could be non-coding sequences and act in the mechanism of small RNA inter- 
ference to suppress the expression of the $c d k$-like loci on 5A and 5D (Al-Kaff et al., 2008). When the expression of Cdk2 in humans, which has the closest homology to the $c d k$ locus in wheat, is disrupted, non-homologous chromosome synapsis at meiosis is observed (Cohen et al., 2006). Conversely, the increase in the expression of the $c d k$-like genes on $5 \mathrm{~A}$ or $5 \mathrm{D}$ in the $P h$ mutant might explain the nature of the Ph1 effects (Al-Kaff et al., 2008). The above facts and the results obtained in this study implied that the rye B chromosome produced certain transcripts acting on the expression of the $c d k$ gene, as the $P h 1$ gene might do. Actually, rye B chromosomes are weakly transcribed (Carchilan et al., 2009).

The effect of the rye B chromosome and its segments on homoeologous pairing was significant as previously reported (Romero and Lacadena, 1980). However, such a drastic increase of chiasma frequency found in the 35+B-9' hybrid (10.23), as compared with that in the 35hybrid (2.78), has not been reported in the previous studies using wheat-rye hybrids containing two doses of rye B chromosomes. This discrepancy might have been caused by the use of double doses of rye $\mathrm{B}$ chromosomes in the previous studies. Whatever the cause is, the increase of chiasma frequency by the monosomic B-9 addition was substantially high for its practical use in inducing crossing over by homoeologous pairing between host and alien chromosomes.

This study was supported by a Grant-in-Aid for Challenging Exploratory Research (No. 23658009) from the Ministry of Education, Culture, Sports, Science and Technology of Japan. Contribution No. 606 from the Laboratory of Plant Genetics, Graduate School of Agriculture, Kyoto University, Japan.

\section{REFERENCES}

Al-Kaff, N., Knight, E., Bertin, I., Foote, T., Hart, N., Griffiths, S., and Moore, G. (2008) Detailed dissection of the chromosomal region containing the $P h 1$ locus in wheat Triticum aestivum: With deletion mutants and expression profiling. Ann. of Bot. 101, 863-872.

Carchilan, M., Kumke, K., Mikolajewski, S., and Houben, A. (2009) Rye B chromosomes are weakly transcribed and might alter the transcriptional activity of A chromosome sequences. Chromosoma 118, 607-616.

Chhuneja, P., Kaur, S., Goel, R. K., Aghaee-Sarbarzeh, M., Prashar, M., and Dhaliwal, H. S. (2008) Transfer of leaf rust and stripe rust resistance from Aegilops umbellulata Zhuk. to bread wheat (Triticum aestivum L.). Genet. Resour. Crop Evol. 55, 849-859.

Cohen, P. E., Pollack, S. E., and Pollard, J. W. (2006) Genetic analysis of chromosome pairing, recombination, and cell cycle control during first meiotic prophase in mammals. Endocr. Rev. 27, 398-426.

Endo, T. R., Nasuda, S., Jones, N., Dou, Q., Akahori, A., Wakimoto, M., Tanaka, H., Niwa, K., and Tsujimoto, H. (2008) Dissection of rye $\mathrm{B}$ chromosomes, and nondisjunction properties of the dissected segments in a common wheat background. Genes Genet. Syst. 83, 23-30.

Feldman, M. (1966) The effect of chromosomes 5B, 5D, and 5A on chromosomal pairing in Triticum Aestivum. Proc. Natl. Acad. Sci. USA 55, 1447-1453.

Gill, B. S., Friebe, B., and Endo, T. R. (1991) Standard karyotype and nomenclature system for description of chromosome bands and structural aberrations in wheat (Triticum aestivum). Genome 34, 830-839.

Griffiths, S., Sharp, R., Foote, T. N., Bertin, I.,Wanous, M., Reader, S., Colas, I., and Moore, G. (2006) Molecular characterization of $P h 1$ as a major chromosome pairing locus in polyploid wheat. Nature 439, 749-752.

Jiang, J., Friebe, B., and Gill, B. S. (1993) Recent advances in alien gene transfer in wheat. Euphytica 73, 199-212.

Jones, R. N., and Puertas, M. J. (1993) The B-chromosomes of rye (Secale cereale L.). In: Frontiers in Plant Science Research (eds.: K. K.Dhir and T. S. Sareen), pp. 81-112. Bhagwati Enterprises, Delhi.

Jones, R. N., and Rees, H. (1969) An anomalous variation due to B chromosomes in rye. Heredity 24, 265-271.

Jones, R. N., González-Sánchez, M., González-García, M., Vega, J. M., and Puertas, M. J. (2008) Chromosomes with a life of their own. Cytogenet. Genome Res. 120, 265-280.

Kang, H., Wang, Y., Fedak, G., Cao, W., Zhang, H., Fan, X., Sha, L., Xu, L., Zheng, Y., and Zhou, Y. (2011) Introgression of chromosome $3 \mathrm{Ns}$ from Psathyrostachys huashanica into wheat specifying resistance to stripe rust. PLoS One 6, $\mathrm{e} 21802$.

Lukaszewski, A. J. (2000) Manipulation of the 1RS. 1BL translocation in wheat by induced homoeologous recombination. Crop Sci. 40, 216-225.

Riley, R., and Chapman, V. (1958) Genetic control of cytologically diploid behavior of hexaploid wheat. Nature 182, 713-715.

Riley, R., Chapman, V., and Kimber, G. (1959) Genetic control of chromosome pairing in intergeneric hybrids with wheat. Nature 183, 1244-1246.

Riley, R., Chapman, V., and Johnson, R. (1968) The incorporation of alien disease resistance in wheat by genetic interference with the regulation of meiotic chromosome synapsis. Genet. Res. Camb. 12, 199-219.

Romero, C., and Lacadena, J. R. (1980) Interaction between rye B-chromosomes and wheat genetic systems controlling homoelogous pairing. Chromosoma 80, 33-48.

Roothaan, M., and Sybenga, J. (1976) No 5-B compensation by rye B-chromosomes. Theor. Appl. Genet. 48, 63-66.

Sakai, K., Nasuda, S., Sato, K., and Endo, T. R. (2009) Dissection of barley chromosome $3 \mathrm{H}$ in common wheat and a comparison of $3 \mathrm{H}$ physical and genetic maps. Genes Genet. Syst. 84, 25-34.

Sandery, M. J., Forster, J. W., Blunden, R., and Jones, R. N. (1990) Identification of a family of repeated sequences on the rye B chromosome. Genome 33, 908-913.

Sears, E. R. (1977) An induced mutant with homoeologous pairing in common wheat. Can. J. Genet. Cytol. 19, 585-593.

Sears, E. R., and Gustafson, J. P. (1993) Use of radiation to transfer alien chromosome segments to wheat. Crop Sci. 33, 897-901.

Viegas, W. S. (1980) The effect of B-chromosomes of rye on the chromosome association in $\mathrm{F}_{1}$ hybrids Triticum aestivum $\mathrm{x}$ Secale cereale in the absence of chromosmes 5B or 5D. Theor. Appl. Genet. 56, 193-198. 